\title{
The Influence of Time Perception on Intertemporal Preference and Its Psychological Mechanism
}

\author{
Shan Jin \\ School of Management, Ji'nan University, Guangzhou, China \\ Email: jsjinaxiao@163.com
}

How to cite this paper: Jin, S. (2020). The Influence of Time Perception on Intertemporal Preference and Its Psychological Mechanism. Open Journal of Social Sciences, 8 , 236-249.

https://doi.org/10.4236/jss.2020.84017

Received: March 5, 2020

Accepted: April 12, 2020

Published: April 15, 2020

Copyright $\odot 2020$ by author(s) and Scientific Research Publishing Inc. This work is licensed under the Creative Commons Attribution International License (CC BY 4.0).

http://creativecommons.org/licenses/by/4.0/

\section{(c) (i) Open Access}

\begin{abstract}
Intertemporal choice refers to people's weighing the costs and benefits at different time points, and then making choices. Because outcomes in such decisions are separated by time, the perception of time should play an important role. Based on previous studies, this paper introduces the influence of time perception differences caused by different time representations, different results and individual differences on intertemporal preference, and explains the causes by perceived-time-based model. In addition, it also introduces the influence of time frame effect, reward and punishment effect, money management guidance, emotion, attention, memory and other cognitive mechanisms on time perception to help people make more rational decisions in the field of Intertemporal choice. In the future, the research direction can be explored from other dimensions of time perception to explore the impact of intertemporal preference and its physiological mechanism.
\end{abstract}

\section{Keywords}

Time Perception, Intertemporal Preference, Perceived-Time-Based Model

\section{Introduction}

Intertemporal choice refers to people's weighing the costs and benefits at different time points, and then making judgments and choices (Frederick, Loewenstein, \& O'Donoghue, 2002). For example, would you like to get 15 yuan today or 20 yuan a month later? Do you usually save or squander money? Do you choose to quit smoking or ignore your health, just want to be happy? Do you hire an experienced employee who can start work immediately, or a talented but inexperienced graduate who needs training? Is it at the expense of resources and envi- 
ronment to develop economy rapidly or to stick to green economy but grow slowly? This kind of choice involves not only the diet and specific economic behavior in the daily life, but also the sustainable development of countries and the formulation of public policies for international organizations to deal with global issues. Therefore, intertemporal choice has been one of the research hotspots in behavioral decision-making and related fields for nearly two decades.

However, most of these studies study the change of subjective value with time from the perspective of value, and ignore the effect of delay time perception on subjective value. Since intertemporal choice is a decision made by considering the two dimensions of time and value, the influence of people's subjective time cognition on intertemporal choice cannot be ignored. Since Takahashi, onono and Radford (2008) found that the relationship between subjective time and objective time is logarithmic function, consumers map objective future time onto subjective perceptions of time is an important driver of intertemporal preferences. Some scholars began to turn their interest to a series of effects on intertemporal choice in the process of human's subjective time perception. Many studies have proved that time perception is an important variable that affects intertemporal preferences.

At present, there is no systematic induction and summary of this research. Based on this, this paper reviews the previous research, summarizes the influence of time perception on intertemporal preferences, explores the interpretation mechanism of the influence of time perception on intertemporal preferences, and on this basis, discusses the current application of time perception in the field of intertemporal choice and the direction worthy of attention in future research.

\section{The Influence of Time Perception on Intertemporal Preference}

Based on previous studies, we will introduce the influence of time perception differences caused by different time representations, different results and individual differences on intertemporal preference. From the perspective of time representation, we will introduce "date/delay effect", "time unpacking effect", "delay/speedup effect", we will also propose "magnitude effect", "sign effect" from the perspective of results and the influence of individual differences on intertemporal preference from the perspective of age and personality traits.

\subsection{The Influence of Different Time Framing on Intertemporal Preference}

Different framing of the same time will have a significant impact on the preference of intertemporal choice, which shows that the discount rate of decision makers will change. According to the research background of time framing, scholars put forward "date/delay effect", "time unpacking effect", "delay/speedup effect". Although the effect is different, in essence, it is the way of time framing that leads to the difference of time perception of decision-makers and affects the 
preference of intertemporal choice.

First of all, the different framing of time units will lead to different perception of time and affect the preference of intertemporal choice. Some scholars have proposed date/delay effect (Read, Frederick, orsel, \& Rahman, 2005; Leboeuf, 2006; DeHart \& Odum, 2015). They pointed out that if the delay in (benefit) results (e.g. after six months) was expressed by date (October 17), the discount rate would be greatly reduced. For example, someone has no obvious preference for "get 1000 yuan today" or "get 1500 yuan after 6 month", but she would prefer "get 1500 yuan on October 17" rather than "get 1000 yuan today". Leboeuf (2006) reached the same conclusion in the experiment. When the time is described by delay rather than by date, the discount rate increases. The main performance of the subjects is that they need to compensate more money when the income is delayed or wait half of the time to get the income, and only a few of them are willing to make long-term investment. The researchers speculate that the representation of time range leads the subjects' attention to the length of time interval, while the representation of date leads the subjects' attention to the time nodes or results. The time length described by date seems shorter than that described by delay in subjective time perception of consumers. Zauberman, Kim, malkoc and Bettman (2009) proved the above viewpoint by experiments. In the experiment, $180 \mathrm{~mm}$ line segments were provided. Half of the subjects estimated the time length of this Monday and next Monday and expressed the perceived subjective time length by line length. The other half estimated the time length from October 24 to November 1 and expressed the perceived subjective time length by line length The results showed that the duration of subjective perception $(M=38.79 \mathrm{~mm})$ in the condition of date representation was significantly shorter than that in the condition of delay representation $\left(M=65.36 \mathrm{~mm}, t_{(26)}=\right.$ 3.09, $P<0.01)$.

Secondly, the expression of time integration or decomposition will also affect the time perception. Liu and Sun (2016) put forward the time unpacking effect, believing that time can be manipulated to affect people's time perception by directly describing the time period. For example, the undivided group is described as from the first week to the third week, while the decomposed group is described as after this week, next week and next week. The research discusses the individual's preference of intertemporal choice under the different time background, and believes that the decomposition description of time period can lengthen people's time perception. Compared with the time integration condition, the subjects under the decomposition condition judge that the time to complete a task is more sufficient, that is, the time perception is longer. Decomposition manipulation lengthen people's subjective perception of time range in larger \& later options and prefers smaller \& sooner options.

Finally, in addition to the above different descriptions of time, the different description of the direction of time will also affect people's subjective time perception, which is mainly manifested in the delay/speedup effect, that is, the discount rate of delay benefit is larger than that of advance benefit (Loewenstein, 
1988). For example, in the case of delay, assume that someone will get 100 yuan in 7 days. Now she has two options: get 100 yuan in 7 days (smaller \& sooner option) and get 150 yuan in 14 days (larger \& later option). Under the condition of advance, she will get 150 yuan in 14 days, and now she faces two options: "get 150 yuan in 14 days" (larger \& later option), or "get 100 yuan in 7 days" (smaller \& sooner option). If she has no obvious preference for SS option and LL option under delay condition, she will prefer LL option under advance condition. When explaining this phenomenon, some scholars think that for the same objective time length, people's perception of waiting time is different under delay condition and advance condition. Their time perception of early acquisition is shorter than that of delayed acquisition, and there is a "delay-advance" framework effect (Li et al., 2014). That is to say, under the condition of advance, people have a shorter sense of 7-day time and a lower discount rate, so they are more inclined to choose the LL option.

\subsection{The Influence of Different Time's Results on Intertemporal Preference}

According to Thaler and Shefrin (1981), it is found that the the reward result is smaller, the the discount rate is higher, and this phenomenon is summed up as the magnitude effect. For example, someone has no obvious preference for the two options of "get 20,000 yuan in one month" and "get 35,000 yuan in three months", but if you reduce the results of these two options by 100 times, she will prefer "get 200 yuan in one month" rather than "get 350 yuan in three months". After that, some scholars use subjective time perception to explain the anomaly, and study the relationship between the amount of the results and the perceived time length. The results show that participants' perception of time duration is affected by the amount of the results, and the large amount of the results leads to a shorter perception of objective time than the small reward result (Wang, Wang, \& Keller, 2015). Previous literature has shown that time perception is a process that can be influenced by attention (Brown, 1985). A large number of results may attract more consumers' attention, resulting in a shorter subjective perception of the objective time length. In addition, the numerical comparison between the amount result and the objective time length may also be one of the reasons for the shortening of people's subjective perception time. The unit dominant model believes that the decision makers will compare the difference between the result dimension and the time dimension. If the difference in money (benefit) is greater than the time dimension, the decision makers will only make decisions on the result dimension, ignoring the time dimension so that the time distance of subjective perception is shorter, and then the option with larger results is selected; on the contrary, if the difference of time is greater than the difference of money, the decision-maker will only make decisions on the time dimension, focusing on the time dimension so that the time distance of subjective perception is longer, and then the option with earlier results is selected (Jiang et al., 2016). 
Secondly, the valence of the decision result of gain or loss will also cause the time perception difference of decision makers. Thaler and Shefrin (1981) found that under the condition of loss, the discount rate is smaller than that under the condition of gain, which means sign effect. For example, if someone has no obvious preference for the options of "get 100 yuan after today" and "get 150 yuan after 7 days", she will prefer "lose 100 yuan after today" rather than "lose 150 yuan after 7 days" after the result of the two options becomes loss. Some scholars think that the subjective time perception is shorter in the loss situation through the research results (Bilgin \& LeBoeuf, 2010). Subjects' perception of the loss results will affect their subjective perception of time. Because the feelings caused by the loss are stronger than the benefits (McGraw et al., 2009), and the events that can cause stronger feelings seem to be closer to the present, so that the subjects' subjective perception time in the loss situation is shorter (Van, Kane, McGraw, \& Dale, 2009). In addition, the loss results can attract more attention than the benefit results, which makes the subjects pay more attention to the results and relatively ignore the time interval, thus leading to the reduction of subjective time. Ruokang Han used psychological time to analyze the time discount under the condition of gain and loss through experiments, and draw a conclusion that the sign effect was caused by the difference between subjective time perception under the condition of gain and loss. That is to say, when the subjects face loss, they think the waiting time is shorter and the discount rate is lower than the benefit condition (Han \& Takahashi, 2012).

\subsection{The Influence of Time Cognitive Differences of Different Individuals on Intertemporal Preference}

In addition to the difference of time cognition caused by the way of time representation, individual age, personality traits will have an impact on time perception and thus affect intertemporal preference.

At present, most studies have found that the discount rate of the elderly is lower than that of the young, that is, the elderly are more willing to wait for delayed benefits (Green, Fry, \& Myerson, 1994; Halfmann, Hedgcock, \& Denburg, 2013; Jimura et al., 2011; Löckenhoff, O’Donoghue, \& Dunning, 2011). For example, Löckenhoff et al. (2011) selected 98 subjects aged 19 - 91 to receive $\$ 5$ immediately and wait for a period of time After 7 days, 30 days, 90 days and 180 days, the reward of other amount (4.75 dollars, 5.25 dollars, 5.5 dollars, 6.5 dollars, 7 dollars, 7.5 dollars) was selected. The results showed that the discount rate decreased with age, that is, the elderly were more willing to wait for larger \& later reward than the young when facing intertemporal choices. Some scholars use subjective time perception to explain the difference of age in intertemporal selection, and find that the subjective perception of time lapse will gradually accelerate with the increase of age. The elderly perceive time lapse faster than the young, so they tend to underestimate time and show more patience (Löckenhoff et al., 2011; Wittmann \& Lehnhoff, 2005); Secondly, individual perception of time length changes with time, which shows that for a given time interval, the 
subjective time perceived by the elderly is shorter than that perceived by the young (Kim \& Zauberman, 2009; Löckenhoff \& Rutt, 2015), no matter the time is shorter (i.e. within hundreds of milliseconds, seconds, minutes) (Turgeon et al., 2016), or when the time length is longer (Wittmann \& Lehnhoff, 2005). The discount rate of the subjective utility of the delayed income is lower, which may lead to that the elderly are more willing to wait for the delayed reward than the young in intertemporal choices. Finally, some studies have found that the perception of time cost in the elderly is usually lower than that in the young. The elderly have more experience in life and more knowledge of the world than the young, so they are more optimistic about the future (Chowdhury, sharot, Wolfe, Düzel, \& Dolan, 2014), and with the decline of perceptual function, the elderly's desire for immediate income and pleasure are often weaker than the young, so the discomfort of waiting for delayed income is weaker (Chowdhury et al., 2013; Eppinger, Nystrom, \& Cohen, 2012). Some studies also believe that the elderly will be better at emotional regulation (Urry \& gross, 2010), so they may be better at regulating the discomfort caused by waiting for delayed earnings. All in all, for the same time delay, the elderly perceive the time faster and shorter than the young, the discount rate of the elderly is lower than that of the young.

Time perception refers to the individual's feeling and judgment on the change of time length and speed without using any timing tools (Huang, 1993). Time perception tendency is a kind of ability gradually developed in people's practical activities, which has individual differences. Wittmann and Paulus (2008) think that the difference of time perception ability may affect the delay discount in intertemporal choices to some extent. The tendency of overestimation of time will lead individuals to choose immediate but smaller benefits, while underestimate of time will make individuals focus on the greater benefits in the future. From the perspective of personality differences, some scholars use simple intertemporal task to investigate the differences between the two groups (time overestimator and time underestimator) by time distance replication task, aiming to explore the influence of time perception on intertemporal decision-making. The results show that the individual's time perception tendency has a significant impact on the intertemporal preference. Compared with the time underestimator, the time overestimator is more inclined to choose the smaller reward that can be realized in real time (Suo et al., 2014). Another study found that there was a significant negative correlation between the degree of impatience and the estimated time interval in the time perception task. The subjects who overestimated the time interval were more reluctant to choose the delayed benefit than those who underestimated the time interval. When choosing between present consumption and future consumption, individuals tend to choose the former (brocas, carrillo, \& tarraso, 2018).

In addition to the differences in age and time perception tendency, time perspective also affects time perception and intertemporal preference. Time perspective refers to the relatively stable psychological and behavioral characteristics of 
individuals' cognition, experience and action on time (Huang et al., 2005). Time perspective reflects people's personality differences in time dimension, mainly divided into past time perspective, present time perspective and future time perspective, which respectively point to individual psychological representation of the past, the present and the future. People's time perspective belongs to relatively stable personality traits. Individuals' time orientations to the past, present and future are different in emphasis. Therefore, time perception may have an impact on intertemporal preference. Simons et al., (2004) believed that future insight is the current expectation of future goals. Individuals with high insight set their goals in the distant future, while individuals with low insight set their goals in the near future. Compared with those who only pay attention to immediate benefits, those who think about the long-term are less likely to have behaviors that are harmful to their long-term health, such as drinking and smoking. Fellows and Farah (2005) think that those who have lower scores in time insight questionnaire have higher discount rate compared with those who have higher scores. Using the classic paradigm of intertemporal selection, examines the intertemporal preference of individuals which divided into "present hedonic" and "future oriented" groups by using Zimbardo Time Perspective Inventory (ZTPI). Compared with the future insight oriented group, the present hedonic oriented group has a higher discount rate and a preference for instant gratification. The researchers believe that the influence of time perspective on intertemporal preference may occur in the evaluation stage (Tao et al., 2015). According to the model based on time perception proposed by Kim and Zauberman (2009), individuals with future orientation focus on the future, and with "future" as the reference point, delay time perception will be shorter. Therefore, the hyperbolic discount decreases more slowly and tends to delay satisfaction. However, present hedonic oriented individuals pay attention to the present, take the "present" as the reference point, and the delay time will be longer subjectively. As a result, the hyperbolic discount decreases rapidly, which shows that the preference for instant gratification.

\section{The Mechanism of the Influence of Time Perception on Intertemporal Preference}

In the previous section, we have introduced the influence of time perception on intertemporal preference from multiple perspectives. Next, we want to explore how time perception affects intertemporal preference in more depth. Different from the previous interpretation models, we want to explain the internal mechanism of time perception influencing intertemporal preference through the model based on subjective time perception.

The time in Du model and hyperbolic discounting is objective time, but when people make intertemporal decision, they usually depend on the subjective time. Recent studies in financial physics, behavioral economics and neuroeconomics fields have focused on the role of subjective time perception in interpreting in- 
tertemporal choice anomalies. As the mathematical analysis of psychophysical laws has become more and more perfect, it is very advantageous to use psychophysical methods to explain the intertemporal preference anomalies (time-based accounts). In psychophysics, Weber-Fechner law has shown that people's inner sense of physical stimulation follows the logarithmic function relationship, while the nonlinear relationship. Takahashi, onono and Radford (2008) found that the hyperbolic model can be derived from the exponential model after the physical time in the Du model is transformed into the mental time by Weber-Fechner law. In addition, the fitting degree between the transformed Du model and the experimental data is better than the simple hyperbolic discounting and exponential model which based on physical time. In addition, it is found that the discount rate calculated by subjective time does not decrease with time. When the subjective time replaces the objective time in Du model, Du model can describe the discount rate than better hyperbolic discounting. Zauberm, Kim, malkoc and Bettman (2009) first verified this relationship by directly measuring subjective time through experiments. In the experiment, the researcher provided $180 \mathrm{~mm}$ line segment, and use the length of the line segment express the perceived subjective time length. The results show that although 12 months is considered to be longer than 6 months subjectively, on average, the subjects think that 12 months is less than twice as long as 6 months, that is to say, the relationship between the objective time length and the measured subjective time length follows Weber-Fechner law, that is, the sensitivity of psychological time decreases, and the subjective estimation of time length is less than the actual objective time length.

Later, scholars found that from this point of view can more accurately describe the behavior of intertemporal choice including the delay effect (i.e. with the increase of delay, the discount rate decreases gradually) and preference reversal. The delay effect refers that the discount rate decreases with the increase of delay. For example, there is no preference difference between the individual who gets 100 yuan today and 1000 yuan one year later or 2000 yuan three years later. According to the hyperbolic discounting, individual time perception completely conforms to the objective time, according to the objective time of 3 years $=1 \times 3$ years, the discount rate after 1 year is calculated as $230 \%$, and the discount rate after 3 years is $100 \%$. The reason of delay effect is that the subjective value of reward decreases with the increase of delay. However, according to the model based on time perception, the individual lacks sensitivity to the objective time, and the subjective perception of the objective time will be shortened, that is, 3 years $=1 \times 1.3$ years. When the subjective time is used to calculate the discount, the discount rate of 1 year and 3 years is $230 \%$. In other words, it is more accurate to use the subjective time to calculate the discount rate to explain the effect (zauberm, Kim, malkoc \& Bettman 2009).

Between the current $\$ 3$ and $\$ 45$ a year later, the subjects tend to choose the former, while between $\$ 30$ a year later and $\$ 45$ two years later, the subjects tend to choose the latter, that is, preference reversal. According to the hyperbolic 
discounting, the farther away the time point from which the event results are obtained, the smaller the discount rate. Therefore, in the face of SS reward and LL reward, compared with LL reward, if people can get small reward immediately, people will choose the former; However, if the time interval of getting small reward is too long, even if the time distance between the two rewards does not change, people will usually choose latter, which leads to preference reversal. According to the model based on subjective time perception, the discount rate of the former is greater than that of the latter, because the time interval of the individual's subjective perception from now to one year is longer than that of the individual's perception from one year to two years. If the discount rate of the LL option which get the rewards after one year is less than that of the SS option which get the reward immediately, the individual tends to choose SS option; However, if the discount rate of the LL option which get the rewards after two years is greater than that of the SS option which get the rewards after one year, the individual tends to choose the LL option, which resulting in preference reversal.

\section{Enlightenment}

Time cognition is an important driving factor of time-related decision-making. We can try to manipulate time perception to influence people's preference in the field of money and health, so that people can choose a more rational and healthy life style.

First of all, in the field of investment and credit, a series of strategies can be adopted to influence intertemporal preference. For example, when providing bonds, monetary investment, or insurance, it is better to emphasize the specific date when they are due to receive profits, because this expression can make the time distance perceived by the decision-maker shorter, reduce the time discount rate and increase the investment willingness. On the contrary, the time information related to loans is better represented in the form of delay, so that the future to the present time interval is longer and repayment is more easy. For example, in the terms of the loan, an extension scheme ("payment after six months") is more attractive than a specific date scheme ("payment by June 2005"). The government can use the date/delay effect to promote saving and stimulate consumption. For example, when calling for savings, it is more effective to "save 10,000 yuan on New Year's Day 2018 and receive 10,360 yuan on New Year's Day 2019," than "save 10,000 yuan on New Year's Day 2018 and receive 10,360 yuan 12 months later". In addition, it has been suggested that the high discount rate of addicts may be attributed to amplified subjective time perception (Zauberman, Kim, malkoc, \& Bettman, 2009). Because the subjective perceived delay time is longer than the actual delay time, the delay option will be given a lower subjective reward value, and thus tend to choose immediate small reward. Previous studies have found evidence of overestimation of delay time in addicts. It is speculated that the impulsive behavior of various pathological brain injury and 
addictive patients, is probably caused by variation of the time perception (Wittmann, Leland, churan, \& Paulus, 2007). Understanding the factors that affect time perception and the role of time perception in intertemporal decision-making is very helpful to promote the healthy behaviors of addicts (smoking, drinking, overeating, pathological gambling, Internet addiction, etc.) and reduce the procrastination behaviors of students and office workers.

Based on the above inference, here are a few tips to help people make rational choices and boost healthy behaviors. First, make good use of time frame effect. Using the date delay effect in the time representation to achieve the purpose that people want longer or shorter time. Taking smoking as an example, when encouraging to quit smoking, we can consider the way of "no smoking from July 12 to July 19 " rather than "no smoking in this week" to dissuade the smokers, because the time interval perceived by the smokers under the condition of date description is shorter, which may be easier to do. The same is true for reducing procrastination. If students are expected to finish their homework as soon as possible or employees are expected to finish their tasks as soon as possible, they can use the date description method to make specific plans, such as setting a deadline to make them feel that the time is urgent and need to speed up.

Second, make good use of the effect of rewards and punishments. When dissuading smokers, try to use the expression of punishment instead of the expression of reward. For example, use a fine of 100 yuan for smoking within a week instead of a reward of 100 yuan for non-smoking within a week, because the discount rate of individuals in the loss situation is lower than that in the gain situation. In the loss situation, smokers may be more likely to persist because they pay more attention to the loss results and thus time perception more shorter.

Third, make good use of the money management guidance. Some researchers put forward money management guidance (ATM) from the perspective of time construction level, which effectively reduces the discount rate of addicts by encouraging subjects to subdivide short-term consumption plans and consider long-term goals when making monthly budget (Black \& Rosen, 2011). By improving the insight ability of addicts and procrastinators in future time, this method can shorten the perception of future time and reduce the discount rate.

Finally, through the cognitive mechanism of emotion, attention and memory, we can improve the ability of time perception so as to make the choice more rational. Studies have shown that substantial waiting time can reduce people's perception of waiting time (Anderson et al., 2012). For example, use music, radio, newspapers and books to enrich the waiting process, reduce the estimation of the duration by distracting the addicts' attention to the time, or improve the addicts' positive emotions and avoid negative emotions by imaging pleasant scenes, so as to shorten the perception waiting time. Some scholars think that by improving the ability of individuals to remember the past events, it is possible to reduce their perception of the waiting time. The discount rate of future can be 
reduced by memory training methods such as digit sequence memory (auditory), digit reverse sequence memory (auditory), word sequence memory (visual), language memory classification (Bickel et al., 2014).

\section{Conclusions}

In addition to some useful suggestions in the previous summary which help people make rational choices and boost healthy behaviors, this paper mainly discussed the relationship between time and intertemporal preference. As we reviewed, early studies treated time as objective information and examined the effect of delays in different lengths and the effect of the location of delays on intertemporal preference. More recently, research has begun to take into account the subjective nature of time and to introduce subjective time as an important explanatory variable for intertemporal preference.

We next discuss some venues for future research. At present, the research of subjective time perception focuses on the estimation of time span, which indirectly affects the intertemporal preference by influencing time span perception. In fact, time perception includes different components such as time length and speed perception, time cost perception, future time perception and related motivation changes (Löckenhoff \& Rutt, 2015), for example, when explaining the influence of different emotions on intertemporal preference, are time cost perception and speed perception more persuasive? Emotion and the passing speed of subjective time influence each other. When you are happy, you will feel that time is passing very fast (Danckert \& Allman, 2005). Conrad et al. (2010) found that when the progress indicator of the task is ahead of the actual progress (i.e. time flies), the experience of the subjects is more pleasant; when the progress indicator is behind (i.e. time slows down), the pleasure of the subjects decreases, and the completion rate of the task decreases. In the future, new research paradigms and methods can be considered to verify that emotion affects the intertemporal preference by influencing the perception of individuals on the speed of time passing. Marmorstein, Grewal and Fishe (1992) found that the emotional experience brought by the price comparison during the shopping process will affect the value estimation of shopping time. The perception of time cost can also be considered in the interpretation of instant gratification. Time prolongation is a loss for individuals, while "instant gratification" is a time gain. Future research needs to further explore the role of time perception in intertemporal selection from other dimensions of time perception, so as to better explain the cognitive mechanism of intertemporal decision-making.

In addition, some researchers have found that time perception has a significant impact on intertemporal choice and the brain regions involved in this process. They believe that the differences in time evaluation also lead to different activities in the parietal lobe, medial temporal lobe, fusiform gyrus, hippocampus and parahippocampal gyrus, thalamus and other brain regions, but the precise functions of these brain regions in intertemporal choice are still unclear. In 
the future, brain imaging technology may be used to investigate the neural mechanism of how time perception plays a role in the process of intertemporal choice.

\section{Conflicts of Interest}

The author declares no conflicts of interest regarding the publication of this paper.

\section{References}

Andersson, P. K., Kristensson, P., Wästlund, E., \& Gustafsson, A. (2012). Let the Music Play or Not: The Influence of Background Music on Consumer Behavior. Journal of Retailing and Consumer Services, 19, 553-560. https://doi.org/10.1016/j.jretconser.2012.06.010

Bickel, W. K., Moody, L., \& Quisenberry, A. (2014). Computerized Working-Memory Training as a Candidate Adjunctive Treatment for Addiction. Alcohol Research: Current Reviews, 36, 123.

Bilgin, B., \& LeBoeuf, R. A. (2010). Looming Losses in Future Time Perception. Journal of Marketing Research, 47, 520-530. https://doi.org/10.1509/jmkr.47.3.520

Black, A. C., \& Rosen, M. I. (2011). A Money Management-Based Substance Use Treatment Increases Valuation of Future Rewards. Addictive Behaviors, 36, 125-128. https://doi.org/10.1016/j.addbeh.2010.08.014

Brown, S. W. (1985). Time Perception and Attention: The Effects of Prospective versus Retrospective Paradigms and Task Demands on Perceived Duration. Perception \& Psychophysics, 3, 115-124. https://doi.org/10.3758/BF03212778

Chowdhury, R., Guitart-Masip, M., Lambert, C., Dayan, P., Huys, Q., Düzel, E., \& Dolan, R. J. (2013). Dopamine Restores Reward Prediction Errors in Old Age. Nature Neuroscience, 16, 648. https://doi.org/10.1038/nn.3364

Chowdhury, R., Sharot, T., Wolfe, T., Düzel, E., \& Dolan, R. J. (2014). Optimistic Update Bias Increases in Older Age. Psychological Medicine, 44, 2003-2012.

https://doi.org/10.1017/S0033291713002602

Conrad, F. G., Couper, M. P., Tourangeau, R., \& Peytchev, A. (2010). The Impact of Progress Indicators on Task Completion. Interacting with Computers, 22, 417-427. https://doi.org/10.1016/j.intcom.2010.03.001

Danckert, J. A., \& Allman, A. A. A. (2005). Time Flies When You're Having Fun: Temporal Estimation and the Experience of Boredom. Brain and Cognition, 59, 236-245. https://doi.org/10.1016/j.bandc.2005.07.002

DeHart, W. B., \& Odum, A. L. (2015). The Effects of the Framing of Time on Delay Discounting. Journal of the Experimental Analysis of Behavior, 103, 10-21. https://doi.org/10.1002/jeab.125

Eppinger, B., Nystrom, L. E., \& Cohen, J. D. (2012). Reduced Sensitivity to Immediate Reward during Decision-Making in Older than Younger Adults. PLoS ONE, 7, e36953. https://doi.org/10.1371/journal.pone.0036953

Fellows, L. K., \& Farah, M. J. (2005). Dissociable Elements of Human Foresight: A Role for the Ventromedial Frontal Lobes in Framing the Future, But Not in Discounting Future Rewards. Neuropsychologia, 43, 1214-1221.

https://doi.org/10.1016/j.neuropsychologia.2004.07.018

Frederick, S., Loewenstein, G., \& O'donoghue, T. (2002). Time Discounting and Time 
Preference: A Critical Review. Journal of Economic Literature, 40, 351-401. https://doi.org/10.1257/jel.40.2.351

Green, L., Fry, A. F., \& Myerson, J. (1994). Discounting of Delayed Rewards: A Life-Span Comparison. Psychological Science, 5, 33-36. https://doi.org/10.1111/j.1467-9280.1994.tb00610.x

Halfmann, K., Hedgcock, W., \& Denburg, N. L. (2013). Age-Related Differences in Discounting Future Gains and Losses. Journal of Neuroscience, Psychology, and Economics, 6, 42. https://doi.org/10.1037/npe0000003

Han, R., \& Takahashi, T. (2012). Psychophysics of Time Perception and Valuation in Temporal Discounting of Gain and Loss. Physica A: Statistical Mechanics and its Applications, 391, 6568-6576. https://doi.org/10.1016/j.physa.2012.07.012

Huang, X. T. (1993). A Cognitive Approach to the Temporal Duration Information Processing. Journal of Southwest China Normal University (Natural Science), 18, 207-215.

Huang, X. T., Zhang, Z. J., Feng, S. H., Guo, X. Y., Lü, H. C., \& Chen, Y. (2005). A New Exploration of Time Psychology. Psychological Science, 28, 1284-1287.

Jiang, C. M., Liu, H. Z., Cai, X. H., \& Li, S. (2016). A Process Test of Priority Models of Intertemporal Choice. Acta Psychologica Sinica, 48, 59-72.

https://doi.org/10.3724/SP.J.1041.2016.00059

Jimura, K., Myerson, J., Hilgard, J., Keighley, J., Braver, T. S., \& Green, L. (2011). Domain Independence and Stability in Young and Older Adults' Discounting of Delayed Rewards. Behavioural Processes, 87, 253-259.

https://doi.org/10.1016/j.beproc.2011.04.006

Kim, B. K., \& Zauberman, G. (2009). Perception of Anticipatory Time in Temporal Discounting. Journal of Neuroscience, Psychology, and Economics, 2, 91. https://doi.org/10.1037/a0017686

LeBoeuf, R. A. (2006). Discount Rates for Time versus Dates: The Sensitivity of Discounting to Time-Interval Description. Journal of Marketing Research, 43, 59-72.

https://doi.org/10.1509/jmkr.43.1.59

Li, A., Zhao, D., Xiong, G., Tan, F., Wang, X., \& Ling, W. (2014). Is Waiting a Kind of Torture? Perceived Waiting Time and the Resulting Irrational Decision Making. Advances in Psychological Science, 22, 1679-1690. https://doi.org/10.3724/SP.J.1042.2014.01679

Liu, Y., \& Sun, Y. (2016). Time Unpacking Effect and Its Impact on Intertemporal Decision Making. Acta Psychologica Sinica, 48, 362-370. https://doi.org/10.3724/SP.J.1041.2016.00362

Löckenhoff, C. E., \& Rutt, J. L. (2015). Age Differences in Time Perception and Their Implications for Decision Making across the Life Span. In Aging and Decision Making (pp. 213-233). London: Academic Press.

https://doi.org/10.1016/B978-0-12-417148-0.00011-X

Löckenhoff, C. E., O’Donoghue, T., \& Dunning, D. (2011). Age Differences in Temporal Discounting: The Role of Dispositional Affect and Anticipated Emotions. Psychology and Aging, 26, 274. https://doi.org/10.1037/a0023280

Loewenstein, G. F. (1988). Frames of Mind in Intertemporal Choice. Management Science, 34, 200-214. https://doi.org/10.1287/mnsc.34.2.200

Marmorstein, H., Grewal, D., \& Fishe, R. P. (1992). The Value of Time Spent in Price-Comparison Shopping: Survey and Experimental Evidence. Journal of Consumer Research, 19, 52-61. https://doi.org/10.1086/209285

Read, D., Frederick, S., Orsel, B., \& Rahman, J. (2005). Four Score and Seven Years from 
Now: The Date/Delay Effect in Temporal Discounting. Management Science, 51, 1326-1335. https://doi.org/10.1287/mnsc.1050.0412

Simons, J., Vansteenkiste, M., Lens, W., \& Lacante, M. (2004). Placing Motivation and Future Time Perspective Theory in a Temporal Perspective. Educational Psychology Review, 16, 121-139. https://doi.org/10.1023/B:EDPR.0000026609.94841.2f

Suo, T., Zhang, F., Zhao, G., \& Li, H. (2014). The Influence of Time Perception Difference on Intertemporal Choice. Acta Psychologica Sinica, 46, 165-173. https://doi.org/10.3724/SP.J.1041.2014.00165

Takahashi, T., Oono, H., \& Radford, M. H. (2008). Psychophysics of Time Perception and Intertemporal Choice Models. Physica A: Statistical Mechanics and Its Applications, 387, 2066-2074. https://doi.org/10.1016/j.physa.2007.11.047

Tao, A., Liu, J., \& Feng, T. (2015). Time Perspective Predicts Delay Discounting. Psychological Science, 38, 279-283.

Thaler, R. H., \& Shefrin, H. M. (1981). An Economic Theory of Self-Control. Journal of Political Economy, 89, 392-406. https://doi.org/10.1086/260971

Turgeon, M., Lustig, C., \& Meck, W. H. (2016). Cognitive Aging and Time Perception: Roles of Bayesian Optimization and Degeneracy. Frontiers in Aging Neuroscience, 8, 102. https://doi.org/10.3389/fnagi.2016.00102

Urry, H. L., \& Gross, J. J. (2010). Emotion Regulation in Older Age. Current Directions in Psychological Science, 19, 352-357. https://doi.org/10.1177/0963721410388395

van Boven, L., Kane, J., McGraw, A. P., \& Dale, J. (2009). Feeling Close: The Emotional Nature of Psychological Distance. ACR North American Advances. https://doi.org/10.2139/ssrn.1531661

Wang, Y., Wang, L., \& Keller, L. R. (2015). Discounting over Subjective Time: Subjective Time Perception Helps Explain Multiple Discounted Utility Anomalies. International Journal of Research in Marketing, 32, 445-448. https://doi.org/10.1016/j.ijresmar.2015.08.006

Wittmann, M., \& Lehnhoff, S. (2005). Age Effects in Perception of Time. Psychological Reports, 97, 921-935. https://doi.org/10.2466/PR0.97.7.921-935

Wittmann, M., \& Paulus, M. P. (2008). Decision Making, Impulsivity and Time Perception. Trends in Cognitive Sciences, 12, 7-12. https://doi.org/10.1016/j.tics.2007.10.004

Wittmann, M., Leland, D. S., Churan, J., \& Paulus, M. P. (2007). Impaired Time Perception and Motor Timing in Stimulant-Dependent Subjects. Drug and Alcohol Dependence, 90, 183-192. https://doi.org/10.1016/j.drugalcdep.2007.03.005

Zauberman, G., Kim, B. K., Malkoc, S. A., \& Bettman, J. R. (2009). Discounting Time and Time Discounting: Subjective Time Perception and Intertemporal Preferences. Journal of Marketing Research, 46, 543-556. https://doi.org/10.1509/jmkr.46.4.543 